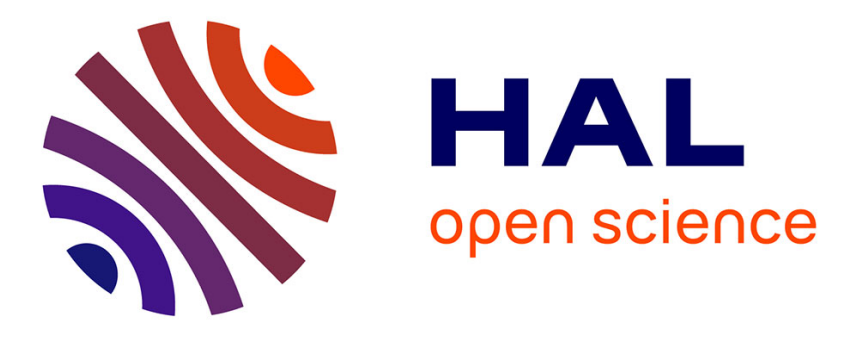

\title{
Observer design for systems with non small and unknown time-varying delay
}

Alexandre Seuret, Thierry Floquet, Jean-Pierre Richard, Sarah K. Spurgeon

\section{To cite this version:}

Alexandre Seuret, Thierry Floquet, Jean-Pierre Richard, Sarah K. Spurgeon. Observer design for systems with non small and unknown time-varying delay. J.J. Loiseau, W. Michiels, S.I. Niculescu, R. Sipahi. Topics in Time-Delay Systems: Analysis, Algorithms and Control, Springer Verlag, pp.233-242, 2009, LNCIS, Lecture Notes in Control and Information Sciences, 978-3-642-02896-0. 10.1007/978-3-642-02897-7_20 . inria-00266457

\section{HAL Id: inria-00266457 https://hal.inria.fr/inria-00266457}

Submitted on 22 Mar 2008

HAL is a multi-disciplinary open access archive for the deposit and dissemination of scientific research documents, whether they are published or not. The documents may come from teaching and research institutions in France or abroad, or from public or private research centers.
L'archive ouverte pluridisciplinaire HAL, est destinée au dépôt et à la diffusion de documents scientifiques de niveau recherche, publiés ou non, émanant des établissements d'enseignement et de recherche français ou étrangers, des laboratoires publics ou privés. 


\title{
Observer design for systems with non small and unknown time-varying delay
}

\author{
Alexandre Seuret ${ }^{1}$, Thierry Floquet ${ }^{1,3}$, Jean-Pierre Richard $^{1,3}$ and Sarah \\ Spurgeon ${ }^{2}{ }^{*}$ \\ 1 LAGIS UMR CNRS 8146, Ecole Centrale de Lille, BP 48, Cité Scientifique, \\ 59651 Villeneuve-d'Ascq, France, \\ seuret.alexandre@ec-lille.fr, Thierry.Floquet@ec-lille.fr, \\ jean-pierre.richard@ec-lille.fr \\ 2 Department of Electronics, University of Kent, UK, S.K.Spurgeon@kent.ac.uk \\ 3 Équipe Projet ALIEN, Centre de Recherche INRIA Lille-Nord Europe, France.
}

Summary. This paper deals with the design of observers for linear systems with unknown, time-varying, but bounded delays (on the state and on the input). In this work, the problem is solved for a class of systems by combining the unknown input observer approach with an adequate choice of a Lyapunov-Krasovskii functional for non small delay systems. This result provides workable conditions in terms of rank assumptions and LMI conditions. The dynamic properties of the observer are also analyzed. A 4th-order example is used to demonstrate the feasibility of the proposed solution.

\subsection{Introduction}

State observation is an important issue for both linear and nonlinear systems. This work considers the observation problem for the case of linear systems with non small and unknown delay. Several authors proposed observers for delay systems (see, e.g., [15, 16]). Most of the literature, as witnessed in [15], considers that the value of the mainly constant delay can be used in the observer realization. This means that the delay is known or measured. Likewise, what are defined as "observers without internal delay" $[3,4,7]$ involve output knowledge both in the present and at delayed instants.

There are currently very few results in which the observer does not assume knowledge of the delay $[2,5,11,18,19]$. These interesting approaches consider linear systems and guarantee an $H_{\infty}$ performance. They are based on stability techniques which are delay independent and lead to the minimization of the state observation error. It is interesting to reduce the probable conservatism

\footnotetext{
* Partially supported by EPSRC grant reference EP/E020763/1, entitled "Robust Output Feedback Sliding Mode Control for Time-delay Systems"
} 
of such results by taking into account information on a delay upper-bound and hence derive an asymptotically stable observer.

In [18], the authors design an observer using a computational delay which can be assimilated to estimation of the delay $\hat{h}$. The conditions which guarantee the convergence of the error dynamics developed for this observer do not take into account the value of $\hat{h}$. This means that the estimate of the state is guaranteed whatever the delay estimate, $\hat{h}$. The property is not related to the conservatism of the conditions. The errors between the real and computational delays are controlled by the discontinuous sliding function. The greater the error between the real and computational delays, the greater the gain of the discontinuous function will be. It is thus straightforward to conclude that a worse estimate of the delay can lead to a high gain in the sliding injection.

In this paper, another method is proposed to solve the problem of the observation of linear systems with unknown time-varying delays which are assumed to be "non small" i.e. the delay function lies in an interval excluding 0 . The result is based on a combination of results on sliding mode observers (see, e.g., $[1,6,8,9,14]$ ) with an appropriate choice of a Lyapunov-Krasovskii functional. For sake of simplicity, the unknown time delay $\tau(t)$ is assumed to be the same for the state and the input. In order to reduce the conservatism of the developed conditions, the existence of known real numbers $d, \tau_{1}$ and $\tau_{2}$ is assumed such that $\forall t \in \mathbb{R}_{+}$:

$$
\begin{gathered}
\tau_{1} \leq \tau(t) \leq \tau_{2} \\
\dot{\tau}(t) \leq d<1 .
\end{gathered}
$$

Here the delay used in the observer is the average of the delay $\left(\tau_{2}+\tau_{1}\right) / 2$. Then the design of the observer does not require the definition or the computation of a delay estimate and the stability conditions only depend on the parameters of the studied system.

Throughout the article, the notation $P>0$ for $P \in \mathbb{R}^{n \times n}$ means that $P$ is a symmetric and positive definite matrix. $\left[A_{1}\left|A_{2}\right| \ldots \mid A_{n}\right]$ is the concatenated matrix with matrices $A_{i} . I_{n}$ represents the $n \times n$ identity matrix.

\subsection{Problem statement}

Consider the linear time-invariant system with state and input delay:

$$
\left\{\begin{array}{l}
\dot{x}(t)=A x(t)+A_{\tau} x(t-\tau(t))+B u(t)+B_{\tau} u(t-\tau(t))+D \zeta(t) \\
y(t)=C x(t) \\
x(s)=\phi(s), \quad \forall s \in\left[-\tau_{2}, 0\right]
\end{array}\right.
$$

where $x \in \mathbb{R}^{n}, u \in \mathbb{R}^{m}$ and $y \in \mathbb{R}^{q}$ are the state vector, the input vector and the measurement vector, respectively. $\zeta \in \mathbb{R}^{r}$ is an unknown and bounded perturbation that satisfies: 


$$
\|\zeta(t)\| \leq \alpha_{1}(t, y, u)
$$

where $\alpha_{1}$ is a known scalar function. $\phi \in C^{0}\left(\left[-\tau_{2}, 0\right], \mathbb{R}^{n}\right)$ is the vector of initial conditions. It is assumed that $A, A_{\tau}, B, B_{\tau}, C$ and $D$ are constant known matrices of appropriate dimensions. The following structural assumptions are required for the design of the observer:

A1. $\operatorname{rank}\left(C\left[A_{\tau}\left|B_{\tau}\right| D\right]\right)=\operatorname{rank}\left(\left[A_{\tau}\left|B_{\tau}\right| D\right]\right) \triangleq p$,

A2. $p<q \leq n$,

A3. The invariant zeros of $\left(A,\left[A_{\tau}\left|B_{\tau}\right| D\right], C\right)$ lie in $\mathbb{C}^{-}$.

Under these assumptions and using the same linear change of coordinates as in [6], Chapter 6, the system can be transformed into:

$$
\left\{\begin{aligned}
\dot{x}_{1}(t)= & A_{11} x_{1}(t)+A_{12} x_{2}(t)+B_{1} u(t) \\
\dot{x}_{2}(t)= & A_{21} x_{1}(t)+A_{22} x_{2}(t)+B_{2} u(t)+D_{1} \zeta(t) \\
& +G_{1} x_{1}(t-\tau(t))+G_{2} x_{2}(t-\tau(t))+G_{u} u(t-\tau(t)) \\
y(t)= & T x_{2}(t)
\end{aligned}\right.
$$

where $x_{1} \in \mathbb{R}^{n-q}, x_{2} \in \mathbb{R}^{q}$ and where $G_{1}, G_{2}, G_{u}, D_{1}$ and $A_{21}$ are defined by:

$$
\begin{gathered}
G_{1}=\left[\begin{array}{c}
0 \\
\bar{G}_{1}
\end{array}\right], G_{2}=\left[\begin{array}{c}
0 \\
\bar{G}_{2}
\end{array}\right], G_{u}=\left[\begin{array}{c}
0 \\
\bar{G}_{u}
\end{array}\right], \\
D_{1}=\left[\begin{array}{c}
0 \\
\bar{D}_{1}
\end{array}\right], A_{21}=\left[\begin{array}{c}
A_{211} \\
A_{212}
\end{array}\right],
\end{gathered}
$$

with $\bar{G}_{1} \in \mathbb{R}^{p \times(n-q)}, \bar{G}_{2} \in \mathbb{R}^{p \times q}, \bar{G}_{u} \in \mathbb{R}^{p \times m}, \bar{D}_{1} \in \mathbb{R}^{p \times r}, A_{211} \in$ $\mathbb{R}^{(q-p) \times(n-q)}, A_{212} \in \mathbb{R}^{p \times(n-q)}$ and $T$ an orthogonal matrix involved in the change of coordinates given in $[6]$.

Under these conditions, the system can be decomposed into two subsystems. $A 1$ implies that the unmeasurable state $x_{1}$ is not affected by the delayed terms and the perturbations. $A 3$ ensures that the pair $\left(A_{11}, A_{211}\right)$ is at least detectable.

In this article, the following lemma will be used:

Lemma 1. [12] For any matrices $A, P_{0}>0$ and $P_{1}>0$, the inequality

$$
A^{T} P_{1} A-P_{0}<0
$$

is equivalent to the existence of a matrix $Y$ such that:

$$
\left[\begin{array}{cc}
-P_{0} & A^{T} Y^{T} \\
Y A & -Y-Y^{T}+P_{1}
\end{array}\right]<0 .
$$




\subsection{Observer design}

Define the following sliding mode observer:

$$
\left\{\begin{aligned}
\dot{\hat{x}}_{1}(t)= & A_{11} \hat{x}_{1}(t)+A_{12} x_{2}(t)+B_{1} u(t) \\
& +\left(L T^{T} G_{l} T-A_{11} L\right)\left(x_{2}(t)-\hat{x}_{2}(t)\right)+L T^{T} \nu(t) \\
\dot{\hat{x}}_{2}(t)= & A_{21} \hat{x}_{1}(t)+A_{22} x_{2}(t)+B_{2} u(t) \\
& +G_{1} \hat{x}_{1}(t-h)+G_{2} x_{2}(t-h)+G_{u} u(t-h) \\
& -T^{T} \nu(t)-\left(A_{21} L+T^{T} G_{l} T\right)\left(x_{2}(t)-\hat{x}_{2}(t)\right) \\
\hat{y}(t)= & T \hat{x}_{2}(t)
\end{aligned}\right.
$$

where the linear gain $G_{l}$ is a Hurwitz matrix and $L$ has the form $[\bar{L} 0]$ with $\bar{L} \in \mathbb{R}^{(n-q) \times(q-p)}$. The computed delay $h=\left(\tau_{2}+\tau_{1}\right) / 2$ is an implemented value that is chosen according to the delay definition. It corresponds to the delay average. The discontinuous injection term $\nu$ is given by:

$$
\nu(t)=\left\{\begin{array}{l}
-\rho(t, y, u) \frac{P_{2}(y(t)-\hat{y}(t))}{\left\|P_{2}(y(t)-\hat{y}(t))\right\|}, \text { if } \quad y(t)-\hat{y}(t) \neq 0 \\
0, \text { otherwise. }
\end{array}\right.
$$

where $P_{2}>0, P_{2} \in \mathbb{R}^{p \times p}$ and where $\rho$ is a nonlinear positive gain yet to be defined. Note that the non delayed terms depending on $x_{2}$ are known because $x_{2}(t)=T^{T} y(t)$. Define $\mu=\left(\tau_{2}-\tau_{1}\right) / 2$.

Remark 1. Compared to [18], this observer does not required an artificial delay $\hat{h}$. It only needs to have an average value of the delay. Contrary to the observer proposed in [18], the implemented delay will appear in the conditions which guarantee stability.

Defining the state estimation errors as $e_{1}=x_{1}(t)-\hat{x}_{1}(t)$ and $e_{2}=x_{2}(t)-$ $\hat{x}_{2}(t)$, one obtains:

$$
\left\{\begin{aligned}
\dot{e}_{1}(t)= & A_{11} e_{1}(t)-L T^{T}\left(G_{l} T e_{2}(t)+\nu(t)\right)+A_{11} L e_{2}(t) \\
\dot{e}_{2}(t)= & A_{21} e_{1}(t)+G_{1} e_{1}(t-\tau(t))+\xi(t)+D_{1} \zeta(t) \\
& +\left(T^{T} G_{l} T+A_{21} L\right) e_{2}(t)+T^{T} \nu
\end{aligned}\right.
$$

where $\xi: \mathbb{R} \longmapsto \mathbb{R}^{p}$ is given by:

$$
\begin{aligned}
\xi(t)= & G_{1}\left(\hat{x}_{1}(t-\tau(t))-\hat{x}_{1}(t-h)\right)+G_{2}\left(x_{2}(t-\tau(t))-x_{2}(t-h)\right) \\
& +G_{u}(u(t-\tau(t))-u(t-h)) .
\end{aligned}
$$

which can be rewritten as:

$$
\xi(t)=\left[\begin{array}{lll}
G_{1} & G_{2} & G_{u}
\end{array}\right] \int_{t-h}^{t-\tau(t)}\left[\begin{array}{c}
\dot{\hat{x}}_{1}(s) \\
\dot{x}_{2}(s) \\
\dot{u}(s)
\end{array}\right] d s .
$$


The function $\xi$ only depends on the known variables $\hat{x}_{1}, x_{2}$ and $u$ and on the unknown delay $\tau(t)$. One can then assume that there exists a known scalar function $\alpha_{2}$ such that:

$$
\|\xi(t)\| \leq \alpha_{2}\left(t, \hat{x}_{1}, x_{2}, u\right) .
$$

Let us define an expression for $\rho$ in (1.6) by using results introduced in the case of control law design [10]. Define $\gamma$, a real positive number and $\rho$ such that:

$$
\rho(t, y, u)=\left\|D_{1}\right\| \alpha_{1}(t, y, u)+\alpha_{2}\left(t, \hat{x}_{1}, x_{2}, u\right)+\gamma .
$$

Introduce the change of coordinates

$$
\left[\begin{array}{l}
\bar{e}_{1} \\
\bar{e}_{2}
\end{array}\right]=T_{L}\left[\begin{array}{l}
e_{1} \\
e_{2}
\end{array}\right]
$$

with

$$
T_{L}=\left[\begin{array}{cc}
I_{n-q} & L \\
0 & T
\end{array}\right]
$$

Using the fact that $L G_{1}=L G_{2}=L G_{u}=L D_{1}=0$, one obtains:

$$
\left\{\begin{aligned}
\dot{\bar{e}}_{1}(t)= & \left(A_{11}+L A_{21}\right) \bar{e}_{1}(t) \\
\dot{\bar{e}}_{2}(t)= & T A_{21} \bar{e}_{1}(t)+T G_{1} \bar{e}_{1}(t-\tau(t))+G_{l} \bar{e}_{2}(t) \\
& -T G_{1} L \bar{e}_{2}(t-\tau(t))+T \xi(t)+T D_{1} \zeta(t)+\nu
\end{aligned}\right.
$$

Theorem 1. Under assumptions $A 1-A 3$ and (1.8) and for all Hurwitz matrices $G_{l}$, system (1.10) is asymptotically stable for any delay $\tau(t)$ in $\left[\begin{array}{ll}\tau_{1} & \tau_{2}\end{array}\right]$ if there exist symmetric definite positive matrices $P_{1}, R_{1}$ and $R_{1 a} \in$ $\mathbb{R}^{(n-q) \times(n-q)}, P_{2} \in \mathbb{R}^{q \times q}$, symmetric matrices $Z_{2}$ and $Z_{2 a} \in \mathbb{R}^{q \times q}$ and a matrix $W \in \mathbb{R}^{(n-q) \times(q-p)}$ such that the following Linear Matrix Inequalities (LMI) hold:

$$
\left[\begin{array}{ccccc}
\Psi_{0} & \Psi_{1} & \Psi_{1} & \Psi_{2} & 0 \\
* & -2 P_{1}+h R_{1} & 0 & 0 & 0 \\
* & * & -2 P_{1}+h R_{1 a} & 0 & 0 \\
* & * & * & \Psi_{3} & -P_{2} T G_{1} \\
* & * & * & * & -P_{1}
\end{array}\right]<0 .
$$

where

$$
\begin{aligned}
& \Psi_{0}=A_{11}^{T} P_{1}+P_{1} A_{11}+A_{211}^{T} W^{T}+W A_{211}, \\
& \Psi_{1}=A_{11}^{T} P_{1}+A_{211}^{T} W^{T}, \\
& \Psi_{2}=\left(A_{21}+G_{1}\right)^{T} T^{T} P_{2}, \\
& \Psi_{3}=G_{l}^{T} P_{2}+P_{2} G_{l}+h Z_{2}+2 \mu Z_{2 a}+R_{2},
\end{aligned}
$$

and 


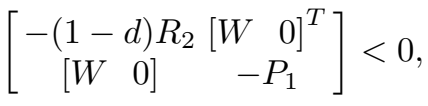

$$
\begin{aligned}
& {\left[\begin{array}{cc}
R_{1} & \left(T G_{1}\right)^{T} P_{2} \\
P_{2} T G_{1} & Z_{2}
\end{array}\right] \geq 0,} \\
& {\left[\begin{array}{cc}
R_{1 a} & \left(T G_{1}\right)^{T} P_{2} \\
P_{2} T G_{1} & Z_{2 a}
\end{array}\right] \geq 0 .}
\end{aligned}
$$

The gain $\bar{L}$ is given by $\bar{L}=P_{1}^{-1} W$.

Proof. Consider the Lyapunov-Krasovskii functional:

$$
\begin{aligned}
V(t)= & \bar{e}_{1}^{T}(t) P_{1} \bar{e}_{1}(t)+\int_{-h}^{0} \int_{t+\theta}^{t} \dot{\bar{e}}_{1}^{T}(s) R_{1} \dot{\bar{e}}_{1}(s) d s d \theta \\
& +\int_{-\mu}^{\mu} \int_{t+\theta}^{t} \dot{\bar{e}}_{1}^{T}(s) R_{1 a} \overline{\bar{e}}_{1}(s) d s d \theta \\
& +\bar{e}_{2}^{T}(t) P_{2} \bar{e}_{2}(t)+\int_{t-\tau(t)}^{t} \bar{e}_{2}^{T}(s) R_{2} \bar{e}_{2}(s) d s .
\end{aligned}
$$

The functional $V$ can be divided into three parts. The first line of (1.13) is designed to control the errors $e_{1}(t)$ subject to the constant delay $h$. The second line presents a functional which takes into account the delay variation around the average delay $h$. The last part which appears in the last line of (1.13) controls the error $e_{2}(t)$.

Using the following transformation

$$
\bar{e}_{i}(t-\tau(t))=\bar{e}_{i}(t)-\int_{t-h}^{t} \dot{\bar{e}}_{i}(s) d s-\int_{t-\tau(t)}^{t-h} \dot{\bar{e}}_{i}(s) d s,
$$

one obtains:

$$
\begin{aligned}
\dot{V}(t)= & \bar{e}_{1}^{T}(t)\left[\left(A_{11}+L A_{21}\right)^{T} P_{1}+P_{1}\left(A_{11}+L A_{21}\right)\right] \bar{e}_{1}(t) \\
& +2 \bar{e}_{2}^{T}(t) P_{2} T\left(A_{21}+G_{1}\right) \bar{e}_{1}(t) \\
& +\bar{e}_{2}^{T}(t)\left[G_{l}^{T} P_{2}+P_{2} G_{l}+R_{2}\right] \bar{e}_{2}(t)-2 \bar{e}_{2}^{T}(t) P_{2} T G_{1} L \bar{e}_{2}(t-\tau(t)) \\
& -(1-\dot{\tau}(t)) \bar{e}_{2}^{T}(t-\tau(t)) R_{2} \bar{e}_{2}(t-\tau(t))+h \dot{e}_{1}^{T}(t) R_{1} \dot{\bar{e}}_{1}(t) \\
& -\int_{t-h}^{t} \dot{\bar{e}}_{1}^{T}(s) R_{1} \dot{\bar{e}}_{1}(s) d s+2 \mu \dot{\bar{e}}_{1}^{T}(t) R_{1 a} \dot{\bar{e}}_{1}(t)-\int_{t-\tau_{1}}^{t-\tau_{2}} \dot{\bar{e}}_{1}^{T}(s) R_{1 a} \dot{\bar{e}}_{1}(s) d s \\
& +\eta_{1}(t)+\eta_{2}(t)+\eta_{3}(t)-2 \rho(t, y, u)\left\|P_{2} \bar{e}_{2}(t)\right\|,
\end{aligned}
$$

where

$$
\begin{aligned}
& \eta_{1}(t)=-2 \bar{e}_{2}^{T}(t) P_{2} T G_{1} \int_{t-h}^{t} \dot{\bar{e}}_{1}(s) d s, \\
& \eta_{2}(t)=-2 \bar{e}_{2}^{T}(t) P_{2} T G_{1} \int_{t-\tau(t)}^{t-h} \dot{\bar{e}}_{1}(s) d s, \\
& \eta_{3}(t)=2 \bar{e}_{2}^{T}(t) P_{2}\left[T D_{1} \zeta(t)+T \xi(t)\right] .
\end{aligned}
$$

The LMI condition (1.12) implies that for any vector $X$ :

$$
X^{T}\left[\begin{array}{cc}
R_{1} & \left(T G_{1}\right)^{T} P_{2} \\
P_{2} T G_{1} & Z_{2}
\end{array}\right] X \geq 0 .
$$

Developing this relation for $X=\left[\begin{array}{c}\dot{\bar{e}}_{1}(s) \\ \bar{e}_{2}(t)\end{array}\right]$, the following inequality holds: 
1 Observer for systems with non small and unknown time-varying delay

$$
-2 \bar{e}_{2}(t) P_{2} G_{1} \dot{\bar{e}}_{1}(s) \leq \bar{e}_{2}(t)^{T} Z_{2} \bar{e}_{2}(t)+\dot{e}_{1}^{T}(s) R_{1} \dot{\bar{e}}_{1}(s) .
$$

Then, integration with respect to $s$ of the previous inequality leads to an upper bound for $\eta_{1}(t)$ :

$$
\begin{aligned}
& \eta_{1}(t) \leq \int_{t-h}^{t} \bar{e}_{2}^{T}(t) Z_{2} \bar{e}_{2}(t) d s+\int_{t-h}^{t} \dot{\bar{e}}_{1}^{T}(s) R_{1} \dot{\bar{e}}_{1}(s) d s, \\
& \eta_{1}(t) \leq h \bar{e}_{2}^{T}(t) Z_{2} \bar{e}_{2}(t)+\int_{t-h}^{t} \dot{\bar{e}}_{1}^{T}(s) R_{1} \dot{\bar{e}}_{1}(s) d s .
\end{aligned}
$$

By using the same techniques, an upper bound for $\eta_{2}$ is found:

$$
\eta_{2}(t) \leq 2 \mu \bar{e}_{2}^{T}(t) Z_{2 a} \bar{e}_{2}(t)+\int_{t-h-\mu}^{t-h+\mu} \dot{\bar{e}}_{1}^{T}(s) R_{1 a} \dot{\bar{e}}_{1}(s) d s .
$$

From (1.9) and from the orthogonality of the matrix $T$, the following inequality holds:

$$
\eta_{3}(t)-2 \rho(t, y, u)\left\|P_{2} \bar{e}_{2}(t)\right\| \leq-2 \gamma\left\|P_{2} \bar{e}_{2}(t)\right\|
$$

Taking into account (1.14), (1.15), (1.16) and the fact that

$$
\dot{\bar{e}}_{1}(t)=\left(A_{11}+\bar{L} A_{211}\right) \bar{e}_{1}(t),
$$

$\dot{V}$ can be upperbounded as follows:

$$
\begin{aligned}
\dot{V}(t) \leq & \bar{e}_{1}^{T}(t)\left(P_{1} \tilde{A}_{11}+\tilde{A}_{11}^{T} P_{1}+h \tilde{A}_{11}^{T} R_{1} \tilde{A}_{11}+2 \mu \tilde{A}_{11}^{T} R_{1 a} \tilde{A}_{11}\right) \bar{e}_{1}(t) \\
& -2 \gamma\left\|P_{2} e_{2}(t)\right\|+\bar{e}_{2}^{T}(t)\left(P_{2} G_{l}+G_{l}^{T} P_{2}+R_{2}+h Z_{2}+2 \mu Z_{2 a}\right) \bar{e}_{2}(t) \\
& +2 \bar{e}_{2}^{T}(t) P_{2}\left(A_{21}+G_{1}\right) \bar{e}_{1}(t) \\
& -(1-\dot{\tau}(t)) \bar{e}_{2}^{T}(t-\tau(t)) R_{2} \bar{e}_{2}(t-\tau(t))-2 \bar{e}_{2}^{T}(t) P_{2} T G_{1} L \bar{e}_{2}(t-\tau(t))
\end{aligned}
$$

where $\tilde{A}_{11}=\left(A_{11}+\bar{L} A_{211}\right)$.

Then, the last term of this inequality can be upperbounded by noting that:

$$
\begin{aligned}
& -2 \bar{e}_{2}^{T}(t) P_{2} T G_{1} L \bar{e}_{2}(t-\tau(t)) \\
& \quad \leq \bar{e}_{2}^{T}(t) P_{2} T G_{1} P_{1}^{-1}\left(T G_{1}\right)^{T} P_{2} \bar{e}_{2}(t)+\bar{e}_{2}^{T}(t-\tau(t)) L^{T} P_{1} L \bar{e}_{2}(t-\tau(t)) \\
& \quad \leq \bar{e}_{2}^{T}(t) P_{2} T G_{1} P_{1}^{-1}\left(T G_{1}\right)^{T} P_{2} \bar{e}_{2}(t)+\bar{e}_{2}^{T}(t-\tau(t))\left(P_{1} L\right)^{T} P_{1}^{-1}\left(P_{1} L\right) \bar{e}_{2}(t-\tau(t)),
\end{aligned}
$$

which leads to the following upperbound:

$$
\begin{aligned}
& \dot{V}(t) \leq\left[\begin{array}{c}
\bar{e}_{1}(t) \\
\bar{e}_{2}(t)
\end{array}\right]^{T} \Psi\left[\begin{array}{l}
\bar{e}_{1}(t) \\
\bar{e}_{2}(t)
\end{array}\right]-2 \gamma\left\|P_{2} \bar{e}_{2}(t)\right\|+\bar{e}_{2}^{T}(t-\tau(t)) \psi_{30} \bar{e}_{2}(t-\tau(t)), \\
& \text { where } \Psi=\left[\begin{array}{cc}
\psi_{10} & \Psi_{2} \\
* & \psi_{20}
\end{array}\right] \text { and : } \\
& \psi_{10}=\left(A_{11}+\bar{L} A_{211}\right)^{T} P_{1}+P_{1}\left(A_{11}+\bar{L} A_{211}\right) \\
& +h\left(A_{11}+\bar{L} A_{211}\right)^{T} R_{1}\left(A_{11}+\bar{L} A_{211}\right) \\
& +2 \mu\left(A_{11}+\bar{L} A_{211}\right)^{T} R_{1 a}\left(A_{11}+\bar{L} A_{211}\right), \\
& \psi_{20}=G_{l}^{T} P_{2}+P_{2} G_{l}+R_{2}+h Z_{2}+2 \mu Z_{2 a} \\
& +P_{2} T G_{1} P_{1}^{-1}\left(T G_{1}\right)^{T} P_{2} \\
& \psi_{30}=(1-d) R_{2}+\left(P_{1}\left[\begin{array}{ll}
\bar{L} & 0
\end{array}\right]\right)^{T} P_{1}^{-1}\left(P_{1}\left[\begin{array}{ll}
\bar{L} & 0
\end{array}\right]\right) .
\end{aligned}
$$


This matrix inequality is not an LMI because of the multiplication of matrix variables. Considering $\psi_{20}$ and $\psi_{30}$, the Schur complement can remove these nonlinearities but for $\psi_{10}$ Lemma 1 is required. As $\psi_{10}$ must be negative definite to have a solution to the problem (1.17), the use of Lemma 1 is possible. Applying it twice to $\psi_{10}$, the nonlinear condition can be expressed as:

$$
\begin{aligned}
& {\left[\begin{array}{ccccc}
\Psi_{0} & \bar{A}_{11}^{T} Y^{T} & \bar{A}_{11}^{T} Y_{a}^{T} & \Psi_{2} & 0 \\
* & \psi_{2} & 0 & 0 & 0 \\
* & * & \psi_{3} & 0 & 0 \\
* & * & * & \Psi_{3} & -P_{2} T G_{1} \\
* & * & * & * & -P_{1}
\end{array}\right]<0 .} \\
& {\left[\begin{array}{cc}
-(1-d) R_{2} & {\left[\begin{array}{c}
\bar{L}^{T} P_{1} \\
0 \\
*
\end{array}\right]} \\
-P_{1}
\end{array}\right]<0 .}
\end{aligned}
$$

where

$$
\begin{aligned}
\bar{A}_{11} & =A_{11}+\bar{L} A_{211} \\
\psi_{2} & =-Y-Y^{T}+h R_{1} \\
\psi_{3} & =-Y_{a}-Y_{a}^{T}+h R_{1 a}
\end{aligned}
$$

Choosing $Y=P_{1}, Y_{a}=P_{1}$ and defining $W=P_{1} \bar{L}$, the LMI conditions from the Theorem appear. Then, if (1.11) and (1.12) are satisfied, (1.19) and (1.20) are also satisfied. Finally the error dynamics are asymptotically stable and converge to the solution $e(t)=0$.

Theorem 1 provides conditions for asymptotic stability of the error dynamics. In the following corollary, it is shown that the error dynamic system is in fact driven to the sliding surface $S_{0}=\left\{\left(\bar{e}_{1}, \bar{e}_{2}\right): \bar{e}_{2}=0\right\}$ in finite time and that a sliding motion is maintained thereafter.

Corollary 1. With the observer gain given in Theorem 1, an ideal sliding motion takes place on $S_{0}$ in finite time.

Proof. Consider the Lyapunov function:

$$
V_{2}(t)=\bar{e}_{2}^{T}(t) P_{2} \bar{e}_{2}(t)
$$

Differentiating (1.21) along the trajectories of (1.10) yields:

$$
\begin{aligned}
\dot{V}_{2}(t)= & \bar{e}_{2}^{T}(t)\left(G_{l}^{T} P_{2}+P_{2} G_{l}\right) \bar{e}_{2}(t)+2 \bar{e}_{2}^{T}(t) P_{2} T\left[T^{T} \nu+A_{21} \bar{e}_{1}(t)\right. \\
& \left.+G_{1} \bar{e}_{1}(t-\tau(t))+G_{1} L \bar{e}_{2}(t-\tau(t))+D_{1} \zeta(t)+\xi(t)\right] .
\end{aligned}
$$

Noting that $G_{l}$ is Hurwitz and using (1.6), the following inequality holds:

$$
\dot{V}_{2}(t) \leq 2\left\|P_{2} \bar{e}_{2}(t)\right\|\left[\left\|A_{21} \bar{e}_{1}(t)+G_{1} \bar{e}_{1}(t-\tau(t))+G_{1} L \bar{e}_{2}(t-\tau(t))\right\|-\gamma\right] .
$$

From Theorem 1 , the errors $\bar{e}_{1}$ and $\bar{e}_{2}$ are asymptotically stable. There thus exist an instant $t_{0}$ and a real positive number $\delta$ such that $\forall t \geq$ $t_{0}, \quad\left\|A_{21} \bar{e}_{1}(t)+G_{1} \bar{e}_{1}(t-\tau(t))+G_{1} L \bar{e}_{2}(t-\tau(t))\right\| \leq \gamma-\delta$. This leads to: 


$$
\begin{aligned}
\forall t \geq t_{0}, \dot{V}_{2}(t) & \leq-2 \delta\left\|P_{2} \bar{e}_{2}(t)\right\| \\
& \leq-2 \delta \sqrt{\lambda_{\min }\left(P_{2}\right)} \sqrt{V_{2}(t)} .
\end{aligned}
$$

where $\lambda_{\min }\left(P_{2}\right)$ is the lowest eigenvalue of $P_{2}$. Integrating the previous inequality shows that a sliding motion takes place on the manifold $S_{0}$ in finite time.

In [18], the observer convergence was improved by enforcing exponential convergence (see $[13,17]$ for definitions). Following the same approach, observer gains can be derived from LMIs in order to ensure the observer error dynamics is similarly exponentially stable in this case.

\subsection{Example}

Consider the system with time-varying delay (1.4) and:

$$
\begin{gathered}
A_{11}=\left[\begin{array}{cc}
0 & 0 \\
0 & -1
\end{array}\right], A_{12}=\left[\begin{array}{cc}
-1 & 0 \\
0 & 0.1
\end{array}\right], \\
A_{21}=\left[\begin{array}{cc}
2 & 3 \\
2 & -1
\end{array}\right], A_{22}=\left[\begin{array}{cc}
-1 & 0 \\
0 & -1
\end{array}\right], \\
G_{1}=\left[\begin{array}{cc}
0 & 0 \\
0.1 & 0.21
\end{array}\right], G_{2}=\left[\begin{array}{cc}
0 & 0 \\
0.2 & 1
\end{array}\right], \\
T=\left[\begin{array}{ll}
1 & 0 \\
0 & 1
\end{array}\right], G_{u}=\left[\begin{array}{l}
0 \\
1
\end{array}\right], D_{1}=B_{1}=B_{2}=\left[\begin{array}{l}
0 \\
0
\end{array}\right],
\end{gathered}
$$

The delay is chosen as $\left.\tau(t)=\tau_{0}+\tau_{1} \sin \left(\omega_{1} t\right)\right)$, with $\tau_{0}=0.225 \mathrm{~s}, \tau_{1}=0.075$ and $\omega_{1}=0.5 s^{-1}$. The control law is

$$
u(t)=u_{0} \sin \left(\omega_{2} t\right)
$$

with $u_{0}=2$ and $\omega_{2}=3$ and the Hurwitz matrix $G_{l}$ is $\left[\begin{array}{cc}-5 & 0 \\ 0 & -3\end{array}\right]$. Using Theorem 1, the following observer gain is obtained:

$$
\bar{L}=\left[\begin{array}{c}
-0.1144 \\
0.0280
\end{array}\right]
$$

Since the system (1.4) is open loop stable, its dynamics are bounded. Thus the function $\alpha_{2}\left(t, \hat{x}_{1}, x_{2}, u\right)$ can be chosen as a constant $K=4$. The simulation results are given in the following figures. Figure 1.1 shows the observation errors. Figures 1.2 and 1.3 show the comparison between the real and observed states.

Figure 1.1 shows that the system enters a sliding motion at time $t=2.8 \mathrm{~s}$. The unmeasured variables converge asymptotically to 0 . 


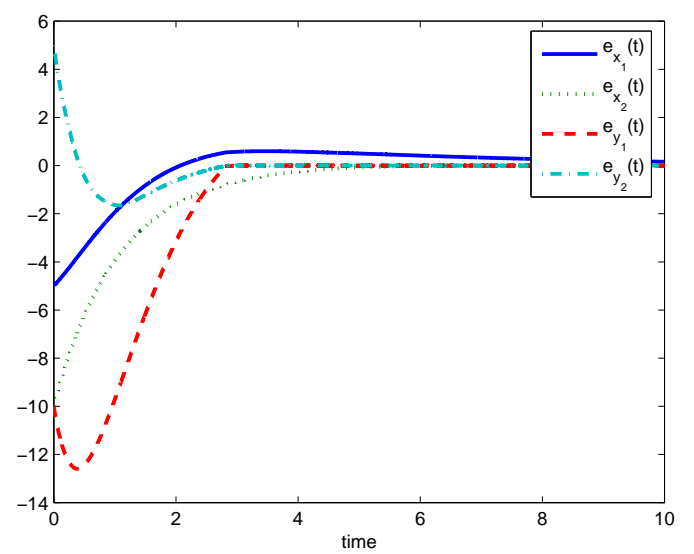

Fig. 1.1. Observation errors for $\tau_{0}=0.225$ and $\tau_{1}=0.075$

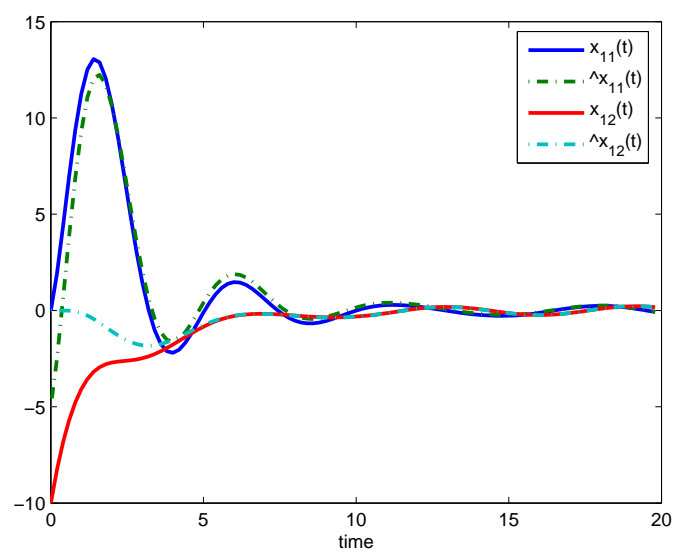

Fig. 1.2. State $x_{1}$ and its estimate $\hat{x}_{1}$

\subsection{Conclusion}

This Chapter has considered the problem of designing observers for linear systems with non small and unknown variable delay on both the input and the state. Delay-dependent LMI conditions have been found to guarantee asymptotic stability of the dynamical error system. The conditions only depend on the delay definition and do not incorporate values of an estimated or computed delay. In addition, the dynamic properties of the proposed observer can be characterized. 


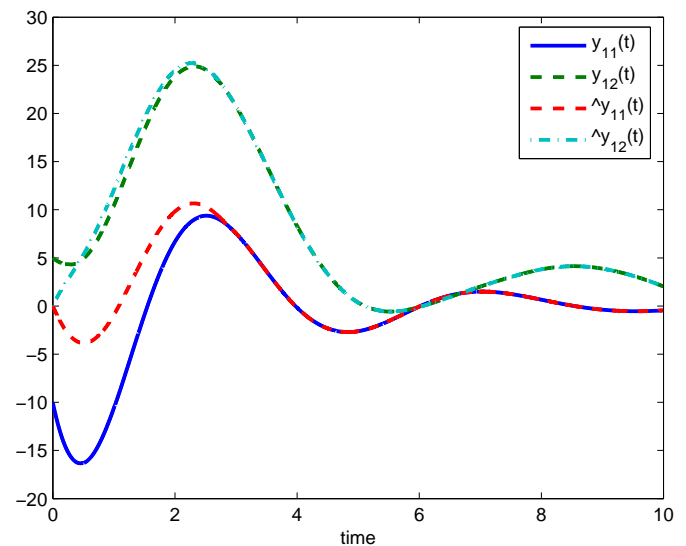

Fig. 1.3. State $x_{2}$ and its estimate $\hat{x}_{2}$

\section{References}

1. J.-P. Barbot, T. Boukhobza and M. Djemaï, "Sliding mode observer for triangular input form", $35^{\text {th }}$ IEEE CDC'g9, Conference on Decision and Control, Kobe, Japan, 1996.

2. H. H. Choi and M. J. Chung, Robust "Observer-based $H_{\infty}$ controller design for linear uncertain time-delay systems", Automatica, Vol. 33, 1997, pp. 1749-1752.

3. M. Darouach, "Linear functional observers for systems with delays in the state variables", IEEE Trans. on Automatic Control, Vol. 46, 2001, pp. 491-497.

4. M. Darouach, P. Pierrot and E. Richard, "Design of reduced-order observers without internal delays", IEEE Trans. on Automatic Control, Vol. 44, 1999, pp. $1711-1713$.

5. C.E. de Souza, R.E. Palhares and P.L.D. Peres, "Robust $H_{\infty}$ filtering for uncertain linear systems with multiple time-varying state: An LMI approach", 38-th IEEE CDC'99, Conference on Decision and Control, Phoenix, AZ, pp. 2023-2028, 1999.

6. Edwards C. and Spurgeon S. K., Sliding Mode Control: Theory and Applications, Taylor \& Francis, 1998.

7. F. W. Fairmar, Kumar and E. Richard, "Design of reduced-order observers without internal delays", IEEE Trans. on Automatic Control, Vol. 44, 1999, pp. 1711-1713.

8. T. Floquet, J.-P. Barbot, W. Perruquetti and M. Djemaï, "On the robust fault detection via a sliding mode disturbance observer", International Journal of control, Vol. 77, 2004, pp. 622-629.

9. T. Floquet, C. Edwards and S. K. Spurgeon, "On sliding mode observers for systems with unknown inputs", International Workshop on Variable Structure Systems, Sardinia, Italy, 2006.

10. E. Fridman, F. Gouaisbaut, M. Dambrine and J.-P. Richard, "Sliding mode control of systems with time-varying delays via a descriptor approach", Int. J. System Sc., Vol. 34, 2003, pp. 553-559. 
11. E. Fridman, U. Shaked and L. Xie, "Robust $H_{\infty}$ Filtering of linear systems with time-varying delay", IEEE Trans. on Automatic Control, Vol. 48, 2003, pp. 159-165.

12. L.-S. Hu, J. Huang and H.-H. Cao, "Robust digital model predicitve control for linear uncertain systems with saturations", IEEE Trans. on Automatic Control, Vol. 49, 2004, pp. 792-796.

13. S-I. Niculescu, C-E. de Souza, L. Dugard and J-M. Dion, "Robust exponential stability of uncertain systems with time-varying delays", IEEE Trans. on Automatic Control, Vol. 43, 1998, pp. 743-748.

14. W. Perruquetti and J. P. Barbot, Sliding Mode Control in Engineering, Ed. Marcel Dekker, 2002.

15. J.-P. Richard, "Time Delay Systems: An overview of some recent advances and open problems", Automatica Vol. 39, 2003, pp. 1667-1694.

16. O. Sename, "New trends in design of observers for time-delay systems", Kybernetica, Vol. 37, 2001, pp. 427-458.

17. A. Seuret, M. Dambrine and J.P. Richard, "Robust exponential stabilization for systems with time-varying delays", 5-th IFAC Workshop on Time-Delay Systems, Leuven, 2004.

18. A. Seuret, T. Floquet, J.P. Richard and S.K. Spurgeon, "A sliding mode observer for linear systems with unknown time varying delay", American Control Conference, New York, USA, 2007.

19. Z. Wang, B. Huang and H. Unbehausen, "Robust $H_{\infty}$ observer design for uncertain time-delay systems:(I) the continuous case", 14-th IFAC World Congress, Beijing, China, pp. 231-236, 1999. 